will greatly facilitate the characterization of possible disease-related methylation patterns on the genomic scale to eventually be used for diagnostic applications.

\section{ACKNOWLEDGMENTS}

This study was supported by grants from the Upper Austrian Government and from the Austrian Federal Ministry for Education, Science, and Culture (bm:bwk) in the context of the Gen-AU project "Ultra-Sensitive Proteomics and Genomics."

\section{COMPETING INTERESTS STATEMENT}

The authors declare no competing interests.

\section{REFERENCES}

1.Jones, P.A. and S.B. Baylin. 2002. The fundamental role of epigenetic events in cancer. Nat. Rev. Genet. 3:415-428.

2.Kow, Y.W. 2002. Repair of deaminated bases in DNA. Free Radic. Biol. Med. 33:886-893.

3.Stacey, K.J., G.R. Young, F. Clark, D.P. Sester, T.L. Roberts, S. Naik, M.J. Sweet, and D.A. Hume. 2003. The molecular basis for the lack of immunostimulatory activity of vertebrate DNA. J. Immunol. 170:3614-3620.

4.Sentis, C., P. Ludena, and J. FernandezPiqueras. 1993. Non-uniform distribution of methylatable CCGG sequences on human chromosomes as shown by in situ methylation. Chromosoma 102:267-271.

5. Bensaada, M., H. Kiefer, G. Tachdjian, J.M. Lapierre, V. Cacheux, A. Niveleau, and P. Metezeau. 1998. Altered patterns of DNA methylation on chromosomes from leukemia cell lines: identification of 5-methylcytosines by indirect immunodetection. Cancer Genet. Cytogenet. 103:101-109.

6.Havlis, J. and M. Trbusek. 2002. 5-Methylcytosine as a marker for the monitoring of DNA methylation. J. Chromatogr. B Analyt. Technol. Biomed. Life Sci. 781:373-392.

7.Robertson, K.D. and A.P. Wolffe. 2000. DNA methylation in health and disease. Nat. Rev. Genet. 1:11-19.

8.Herman, J.G. and S.B. Baylin. 2003. Gene silencing in cancer in association with promoter hypermethylation. N. Engl. J. Med. 349:2042-2054.

9.Wicki, R., C. Franz, F.A. Scholl, C.W. Heizmann, and B.W. Schafer. 1997. Repression of the candidate tumor suppressor gene $\mathrm{S} 100 \mathrm{~A} 2$ in breast cancer is mediated by site-specific hypermethylation. Cell Calcium 22:243-254.

10.Bernardino, J., M. Lombard, A. Niveleau, and B. Dutrillaux. 2000. Common methylation characteristics of sex chromosomes in somatic and germ cells from mouse, lemur and human. Chromosome Res. 8:513-525.

11.Bernardino-Sgherri, J. and B. Dutrillaux. 2001. Compaction, stainability and methylation of the late replicating $\mathrm{X}$ chromosome in mouse female fibroblasts. Cytogenet. Cell Genet. 94:79-81.

12.Drets, M.E. and M.W. Shaw. 1971. Specific banding patterns of human chromosomes. Proc. Natl. Acad. Sci. USA 68:2073-2077.

13.Nichol, K. and C.E. Pearson. 2002. CpG methylation modifies the genetic stability of cloned repeat sequences. Genome Res. 12:1246-1256.

14.Tuck-Muller, C.M., A. Narayan, F. Tsien, D.F. Smeets, J. Sawyer, E.S. Fiala, O.S. Sohn, and M. Ehrlich. 2000. DNA hy- pomethylation and unusual chromosome instability in cell lines from ICF syndrome patients. Cytogenet. Cell Genet. 89:121-128.

15.Schmutte, C. and R. Fishel. 1999. Genomic instability: first step to carcinogenesis. Anticancer Res. 19:4665-4696.

Received 23 November 2004; accepted 14 January 2005.

Address correspondence to Christian Wechselberger, Upper Austrian Research $\mathrm{GmbH}$, Center for Biomedical Nanotechnology, Scharitzerstr. 6-8, 4020 Linz, Austria. e-mail: christian.wechselberger@uar.at

\title{
Shuttle system allowing simplified cloning of expression cassettes into advanced generation lentiviral vectors
}

Ina Gruh, Kristin Schwanke, Stephanie Wunderlich, Ulrike Blömer, Michaela Scherr, Arnold Ganser, Axel Haverich, and Ulrich Martin

Leibniz Research Laboratories, Hannover, Germany

BioTechniques 38:530-534 (April 2005)

Recent lentiviral vector types have shown highly efficient stable transgene expression in terminally differentiated cells $(1,2)$. Despite major advantages of those vectors, lentiviral vector production and gene transfer remains labor-intensive and time-consuming and therefore not appropriate as the standard gene transfer approach for many laboratories.

Large vector size, resulting in low cloning efficiencies, and the absence of convenient multicloning sites leads to complex cloning strategies. In addition, during the cloning of full-length viral plasmid clones, homologous recombination between long terminal repeat (LTR) regions is frequently observed $(3,4)$ even in recA, recB, recJ, and $s b c C$ mutant bacterial strains and often results in the loss of the entire viral sequence except a single copy of an LTR (4).
Here we describe a novel lentiviral cloning system, LentiShuttle, which allows for fast and convenient generation of lentiviral vectors with different expression cassettes. The whole expression cassette is constructed within a high-copy plasmid with convenient multicloning sites. Finally, the cassette is transferred into the lentiviral vector via a simple restriction digest and standard sticky end ligation reaction. All cloning steps prior to the final transfer into pLentiShuttle, including those in pBShuttle, can be carried out in standard Escherichia coli strains such as TOP10 (Invitrogen, Karlsruhe, Germany), which can easily be prepared in the laboratory. Only for the very last cloning step, the insertion of complete expression cassettes between the viral LTRs of pLentiShuttle, is it recommended to use special (usually expensive) 
commercially available recombination-deficient $E$. coli strains, such as Max Efficiency Stb12, Stbl3, or ElectroMax Stbl4 (all from Invitrogen). Other commonly used recombinations impeding E. coli strains, such as HB101 (Takara, Otsu, Japan), are less efficient in preventing recombinations of lentiviral transfer vectors during cloning (4).

The lentiviral cloning system LentiShuttle, comprising the highcopy shuttle plasmid pBShuttle (Figure 1A), and the lentiviral vector pLentiShuttle (Figure 1B) is based on the plasmids pBluescript ${ }^{\circledR}$ II SK(-) (Stratagene, La Jolla, CA, USA) and pShuttle (BD Biosciences, Heidelberg, Germany) and the self-inactivating (SIN) lentiviral transfer plasmid pHR'SINcPPTCEW (5). pLentiShuttle contains all the elements of an advanced generation lentiviral transfer vector, including LTRs, but no expression cassette. In order to generate pLentiShuttle, recognition sequences for the homing endonucleases I-Ceu I and PI-Sce I $(6,7)$ (see Figure 1C) were inserted between the LTRs of the pHR'SINcPPT-CEW backbone and in pBluescript II SK(-) to construct pBShuttle. Both recognition sequences are very long (26 and $39 \mathrm{bp}$ ) and are not present in viral or vertebrate genomic sequences or in standard

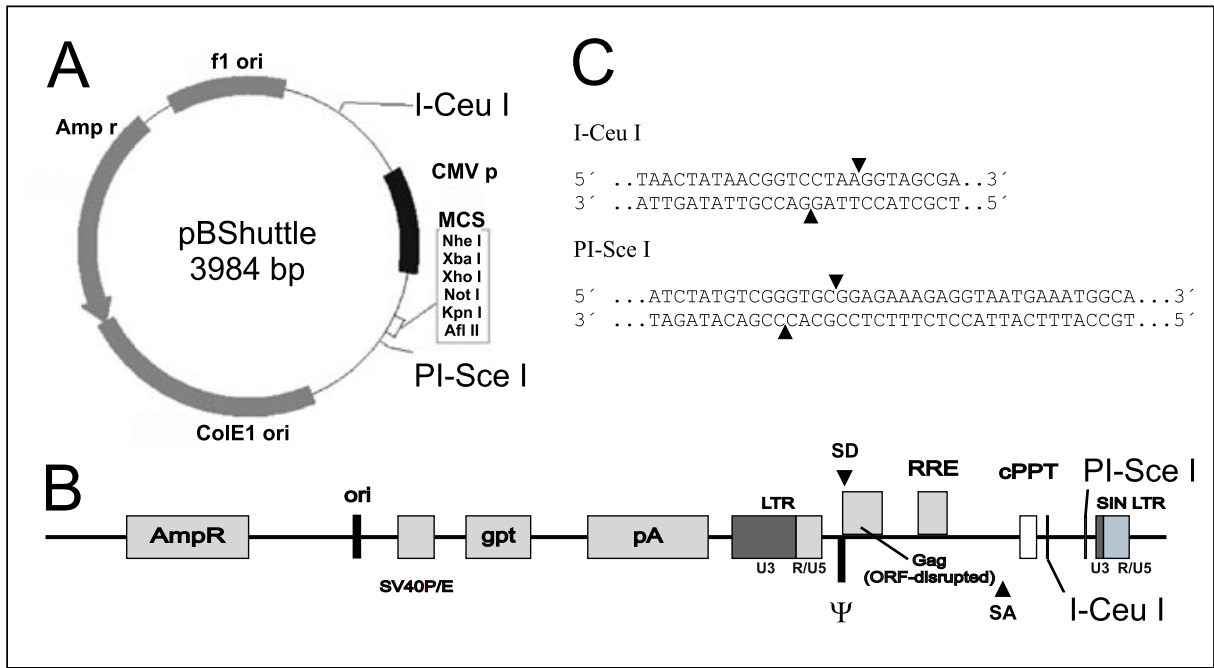

Figure 1. Design of the LentiShuttle vector system. High-copy plasmid pBShuttle with I-Ceu I and PI-Sce I sites enclosing a multicloning site (A) allows for subcloning of expression cassettes between the LTRs of the lentiviral transfer plasmid pLentiShuttle $(+)(B)$, containing recognition sequences for homing endonucleases I-Ceu I and PI-Sce I (C). AmpR, ampicillin resistance gene; cPPT, central polypurine tract; gpt: guanine phosphoribosyl transferase gene; LTR, long terminal repeat; ori, origin of replication; pA, polyadenylation signal; $\Psi$, packaging signal; RRE, rev responsive element; SA, splice acceptor site; SD, splice donor site; SIN LTR, self-inactivating LTR; SV40P/E: simian virus 40 promotor/enhancer.

I-Ceu I and PI-Sce I (New England Biolabs, Beverly, MA, USA) into the lentiviral plasmid pLentiShuttle. For virus production, HEK293T cells were transfected with the lentivirus transfer constructs, the packaging plasmid pCMV $\Delta$ R8.91, and the VSV$\mathrm{G}$ envelope-coding plasmid pMD.G $(1,8)$. After $48 \mathrm{~h}$, the virus-containing medium was collected. Biologic titration of nonconcentrated supernatants was performed on HEK293T cells (8). After $72 \mathrm{~h}$, the proportion of GFPpos to GFP ${ }^{\text {neg }}$ or RedStarpos to RedStar ${ }^{\text {neg }}$ cells was determined by flowcytometry. Titers of infectious units (IU) were back-calculated to the initial cell number $\left(1 \times 10^{5} /\right.$ well $)$ and $1 \mathrm{~mL}$ of nonconcentrated vector supernatant (Figure 2A). Details on virus production and analysis are available online as supplementary material on the BioTechniques' web site at Www. BioTechniques.com.

plasmid vectors. Details on the construction of the vectors are available online as supplementary material on the BioTechniques' web site at www.BioTechniques.com.

By using this system, we were able to clone different lentiviral transfer vectors mediating expression of red and green fluorescent proteins (GFPs) or antibiotic resistances under the control of several promoters/enhancers. A promoterless construct was included in order to rule out expression from human immunodeficiency virus (HIV)derived regulatory sequences.

Expression cassettes were generated in pBShuttle constructs and subcloned with

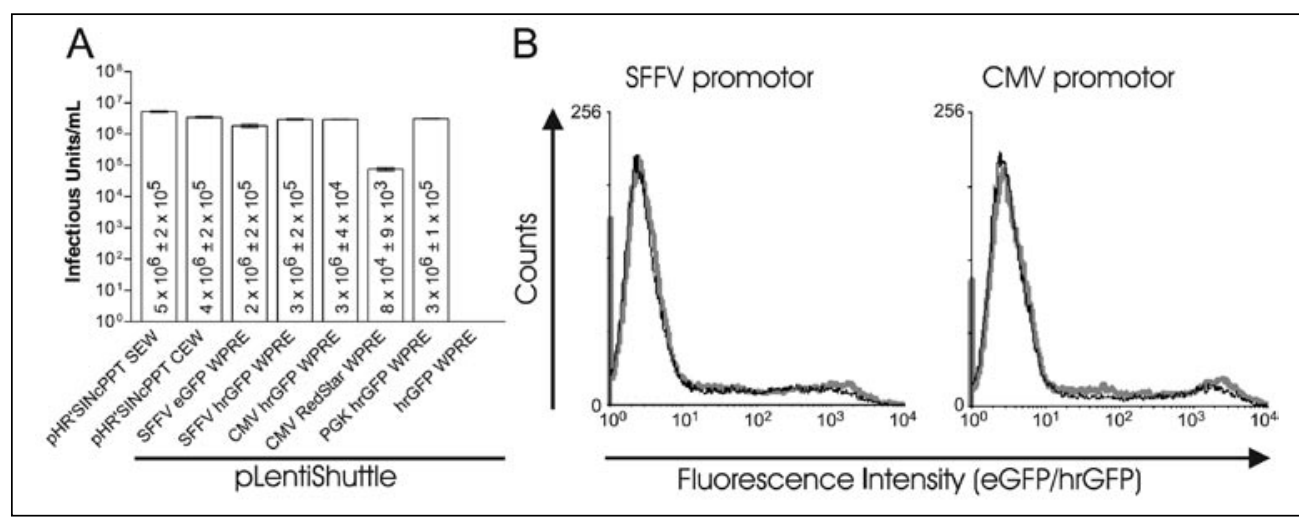

Figure 2. Infectious titers and expression levels obtained with LentiShuttle constructs are on a similar level than for conventional advanced generation lentiviral vectors. Infectious titers of nonconcentrated cell culture supernatants have been determined for all constructs in parallel by fluorescence-activated cell sorter (FACS) analysis of one experiment performed in duplicate. Error bars represent standard deviation (A). Green fluorescent protein (GFP) expression levels in HEK293T cells conferred by conventional advanced generation lentiviral vectors (pHR'SINcPPT SFFV EGFP WPRE, pHR'SINcPPT CMV EGFP WPRE, bold grey lines) and pLentiShuttle constructs (pLentiShuttle SFFV hrGFP WPRE, pLentiShuttle CMV hrGFP WPRE, black lines) have been determined by FACS analysis after transduction using a multiplicity of infection (MOI) of 1 (B). SFFV, spleen focus-forming virus; EGFP, enhanced GFP; CMV, cytomegalovirus. 
Viral vector titers and expression levels obtained with pLentiShuttle constructs are absolutely comparable to those of conventional lentiviral vectors containing similar expression cassettes. Direct comparison of conventional advanced generation lentiviral vectors comprising enhanced GFP (EGFP) under the control of cytomegalovirus (CMV) or spleen focus-forming virus (SFFV) promotor, with the corresponding LentiShuttle vectors and LentiShuttle vectors with phosphoglycerate kinase (PGK) promotor or the humanized Renilla reniformis GFP (hrGFP) instead of EGFP, revealed similar packaging efficiencies (Figure 2A). The actual titer of pLentiShuttle CMV RedStar WPRE may be higher than determined by flow cytometry, as the wavelength applied for excitation during flow cytometry (standard argon laser, wavelength $488 \mathrm{~nm}$ ) does not match the excitation peak of the DsRed variant RedStar (excitation maximum, $558 \mathrm{~nm}$ ) (9). A helium-neon laser (wavelength $543 \mathrm{~nm}$ ) for excitation and special filters should be used for optimal analysis of RedStar emmission. No GFP positive cells were detected for the control plasmid pLentiShuttle hrGFP WPRE (Figure 2A), demonstrating that expression of transgenes exclusively depends on the promoters inserted in our vectors. To exclude the possibility that the negative control virus preparation did not contain infectious virus, infectious titers of all viruses were determined from cell lysates of infected HEK293T cells using a real-time PCR-based assay (10) (data not shown).

We were able to show that transduction with pLentiShuttle vectors resulted in expression levels comparable to conventional lentiviral vectors (Figure 2B). In conclusion, our novel lentiviral cloning system LentiShuttle allows simplified cloning and represents an easy-to-use advanced generation lentiviral vector system with viral titers and expression levels comparable to those of conventional lentiviral vectors. As cloning problems differ case-by-case, it is difficult to quantify the time saved during cloning compared to conventional lentiviral vectors. However, according to our experience, LentiShuttle drastically reduces cloning problems, as well as the average duration and costs of cloning procedures.

\section{ACKNOWLEDGMENTS}

This work was supported by the HILF program of the Hannover Medical School, the Niedersachsen Stiftung/ CORTISS and by the Aventis Foundation. We gratefully acknowledge the excellent technical assistance of Karin Battmer (Hannover Medical School). pHR'SIN-CE, $p C M V \Delta R 8.91$, and $p M D$. $G$ were kindly provided by Didier Trono (University of Geneva), pHR'SINSEW was a gift of Manuel Grez (Georg Speyer Haus Frankfurt). The plasmid pGK-loxP was provided by Francis Stewart (Biotec, TU Dresden), and the improved DsRed variant, RedStar, was provided by Michael Knop (EMBL Heidelberg). We are grateful to Andreas Rohde (University of Cologne) for revising the manuscript.

\section{COMPETING INTERESTS STATEMENT}

The authors declare no competing interests.

\section{REFERENCES}

1.Naldini, L., U. Blomer, F.H. Gage, D. Trono, and I.M. Verma. 1996. Efficient transfer, integration, and sustained long-term expression of the transgene in adult rat brains injected with a lentiviral vector. Proc. Natl. Acad. Sci. USA 93:11382-11388.

2.Kafri, T., U. Blomer, D.A. Peterson, F.H. Gage, and I.M. Verma. 1997. Sustained expression of genes delivered directly into liver and muscle by lentiviral vectors. Nat. Genet. 17:314-317.

3.Peden, K.W. 1992. Instability of HIV sequences in high copy number plasmids. J. Acquir. Immune Defic. Syndr. 5:313-315.

4.Yamada, K., H. Morozumi, and T. Okamoto. 1995. LTR-directed homologous recombination of full-length HIV-1 provirus clone in recA(-) bacteria. Arch. Virol. 140:1007-1014.

5.Demaison, C., K. Parsley, G. Brouns, M. Scherr, K. Battmer, C. Kinnon, M. Grez, and A.J. Thrasher. 2002. High-level transduction and gene expression in hematopoietic repopulating cells using a human imunodeficiency virus type 1-based lentiviral vector containing an internal spleen focus forming virus promoter. Hum. Gene Ther. 13:803-813.

6.Bremer, M.C., F.S. Gimble, J. Thorner, and C.L. Smith. 1992. VDE endonuclease cleaves
Saccharomyces cerevisiae genomic DNA at a single site: physical mapping of the VMA1 gene. Nucleic Acids Res. 20:5484.

7.Marshall, P. and C. Lemieux. 1992. The ICeuI endonuclease recognizes a sequence of 19 base pairs and preferentially cleaves the coding strand of the Chlamydomonas moewusii chloroplast large subunit rRNA gene. $\mathrm{Nu}$ cleic Acids Res. 20:6401-6407.

8.Blomer, U., I. Gruh, H. Witschel, A. Haverich, and U. Martin. 2005. Shuttle of lentiviral vectors via transplanted cells in vivo. Gene Ther. 12:67-74.

9.Knop, M., F. Barr, C.G. Riedel, T. Heckel, and C. Reichel. 2002. Improved version of the red fluorescent protein (drFP583/DsRed/ RFP). BioTechniques 33:592-598.

10.Scherr, M., K. Battmer, U. Blomer, A. Ganser, and M. Grez. 2001. Quantitative determination of lentiviral vector particle numbers by real-time PCR. BioTechniques 31:520-524.

Received 16 November 2004; accepted 2 February 2005.

Address correspondence to Ulrich Martin, Leibniz Research Laboratories, Podbielskistr. 380, 30659 Hannover, Germany. e-mail:martin.ulrich@mh-hannover.de 